\title{
Vortices in magnetically coupled superconducting layered systems
}

\author{
Roman G. Mints \\ School of Physics and Astronomy, Raymond and Beverly Sackler Faculty of Exact Sciences, \\ Tel Aviv University, Tel Aviv 69978, Israel \\ Vladimir G. Kogan and John R. Clem \\ Ames Laboratory - DOE and Department of Physics and Astronomy, Iowa State University, Ames Iowa 50011
}

(December 2, 2017)

\begin{abstract}
Pancake vortices in stacks of thin superconducting films or layers are considered. It is stressed that in the absence of Josephson coupling topological restrictions upon possible configurations of vortices are removed and various examples of structures forbidden in bulk superconductors are given. In particular, it is shown that vortices may skip surface layers in samples of less than a certain size $R_{c}$ which might be macroscopic. The Josephson coupling suppresses $R_{c}$ estimates.
\end{abstract}

PACS 74.60.-w, 74.80.Dm

\section{INTRODUCTION}

Attempts to measure the anisotropy parameter in Biand Tl-based high- $T_{c}$ superconducting compounds yield very large values $\sim 100-300.1$ In some layered organic superconductors this parameter is even higher. 2 This has led many to believe that the Josephson coupling in these materials is so weak that for many purposes it can be disregarded altogether. Models of vortices in these compounds based on pure magnetic coupling between twodimensional mancake vortices proved to suffice for many applications. 3

We consider in this paper a system of thin superconducting layers coupled only via the magnetic field between them. This system is qualitatively different from the bulk superconductors: there is no phase coherence across the layers. In three-dimensional bulk materials vortices are banned from terminating inside superconductor because of topology: the phase changes by $2 \pi$ when one circles the vortex core at which the phase is singular. A core termination would have meant that the phase could acquire the $2 \pi$ change along a path that does not contain a singularity.

Clearly, this ban does not hold in layered systems with no Josephson coupling. A vortex perpendicular to the layers or, better to say, a stack of pancake vortices (or "pancakes"), may terminate, ${ }^{1}$ in principle, at any layer and channel the flux at least partially into interlayer space. This event might be energetically unlikely, but it is not forbidden by topology of the phase, which is defined separately in each layer.

We argue below that within the model of layered ma-

\footnotetext{
${ }^{1}$ We use the term "vortex termination in the layer $\mathrm{N}$ " for the situation when the vortex cores are present in the layer $\mathrm{N}$ and in all layers under, whereas the cores are absent in the layers above $\mathrm{N}$ up to the the sample surface.
}

terials with zero Josephson coupling such termination in layers adjacent to the surface might be energetically favorable in samples of finite size. We use the formal technique suggested by one of us, 3 which is reviewed briefly. The technique allows us to compare energies of various configurations in a straightforward manner. As one of the applications, we compare energies of two configurations: one with a vortex piercing all layers of a half-space multilayer and another with the vortex terminating in one of the top layers. We show that the energy cost of the subsurface vortex termination diverges with the sample size $R$ :

$$
\Delta \epsilon \sim \frac{\phi_{0}^{2}}{16 \pi^{2} \Lambda} \ln \frac{R}{\kappa \lambda_{a b}}
$$

where $\Lambda=\lambda_{a b}^{2} / s, \lambda_{a b}$ is the penetration depth, $s$ is the layer periodicity, $\kappa=\lambda_{a b} / \xi_{a b}$, and $\xi_{a b}$ is the coherence length. The divergence of $\Delta \epsilon$ is weak, however, and in samples smaller than $R_{c} \sim \kappa \lambda_{a b}$ the subsurface termination becomes energetically favorable. With $\lambda_{a b} \approx 0.3 \mu \mathrm{m}$, $\kappa \approx 100$ for Bi-2212, we estimate $R_{c} \sim 30 \mu \mathrm{m}$ with a large numerical factor so that $R_{c}$ may reach a macroscopic size.

This estimate is reduced considerably by including the Josephson coupling because one has to include in the balance the energy of Josephson strings channelling the flux sideways into the interlayer space. We estimate, however, that even then $R_{c}$ remains on the order of microns. This suggests that if the surface of a layered compound like Bi-2212 has defects separated by $R_{c}$, the occurrence of vortex cores at the surface is a rare event. We also show that for a vortex terminating at a depth $\ll \lambda_{a b}$ under the surface, most of the magnetic flux crosses the corefree layers into the free space, the absence of the core 
notwithstanding. Hence, we expect vortices terminating under the surface to be invisible for the scanning tunneling technique, but detectable in decoration experiments.

Other examples of possible but unusual configurations of pancake vortices in layered materials are considered. It is shown that a vortex in a film of a finite size and containing several superconducting layers may have normal cores only in internal layers and carry flux different from the flux quantum $\phi_{0}$. The last feature appears also in exotic configurations such as a stack of pancake vortices situated in every other layer. Implications of these possibilities are discussed.

\section{APPROACH}

We begin with a brief review of thin superconducting films. As was stressed by Pearl, 10 the situation in a thin film differs from that of a bulk since a large contribution to the energy of a vortex comes from the stray fields. In fact, the problem of a vortex in a thin film is reduced to that of the field distribution in free space subject to certain boundary conditions at the film surface. Since $\operatorname{curl} \mathbf{h}=\operatorname{div} \mathbf{h}=0$ outside, one can introduce a scalar potential for the outside field:

$$
\mathbf{h}=\nabla \varphi, \quad \nabla^{2} \varphi=0
$$

To formulate the boundary conditions for the outside Laplace problem let us consider a film of thickness $d \ll \lambda$ occupying the $x y$ plane; $\lambda$ is the bulk penetration depth of the film material. For a vortex at $\mathbf{r}=0$, the London equations for the film interior read:

$$
\mathbf{h}+\frac{4 \pi \lambda^{2}}{c} \operatorname{curl} \mathbf{j}=\phi_{0} \hat{\mathbf{z}} \delta(\mathbf{r})
$$

where $\hat{\mathbf{z}}$ is the unit vector along the vortex axis. Averaging over the thickness $d$ we obtain

$$
h_{z}+\frac{4 \pi \Lambda}{c} \operatorname{curl}_{z} \mathbf{g}=\phi_{0} \delta(\mathbf{r})
$$

where $\mathbf{g}(\mathbf{r})$ is the sheet current density, $\mathbf{r}=(x, y)$, and $\Lambda=\lambda^{2} / d$ is the film penetration depth. Other components of London equation turn identities after averaging.

Since all derivatives $\partial / \partial z$ are large relative to the tangential $\partial / \partial \mathbf{r}$, the Maxwell equation $\operatorname{curl} \mathbf{h}=4 \pi \mathbf{j} / c$ is reduced to conditions relating the sheet current to discontinuities of the tangential field:

$$
\frac{4 \pi}{c} g_{x}=h_{y}^{-}-h_{y}^{+}, \quad \frac{4 \pi}{c} g_{y}=h_{x}^{+}-h_{x}^{-} .
$$

Here the superscripts \pm stand for the upper and lower $(z= \pm d / 2)$ faces of the film. The field component perpendicular to the film, $h_{z}$, is the same at both film faces.

Substituting Eq. (5) into Eq. (4) and using $\operatorname{div} \mathbf{h}=0$, we obtain:

$$
h_{z}+\Lambda\left(\frac{\partial h_{z}^{-}}{\partial z}-\frac{\partial h_{z}^{+}}{\partial z}\right)=\phi_{0} \delta(\mathbf{r})
$$

This equation along with

$$
h_{z}^{+}=h_{z}^{-}
$$

and conditions at infinity constitute the boundary conditions for the Laplace problem, Eq. (2), of the field distribution outside the film.

Let us turn to the question of energy. We consider a general situation of vortices in finite bulk samples. The energy consists of the London energy (magnetic + kinetic) inside the sample, $\epsilon^{(i)}$, and the magnetic energy outside, $\epsilon^{(a)}$ :

$$
\epsilon^{(i)}=\int \frac{d V}{8 \pi}\left[h^{2}+\left(\frac{4 \pi \lambda}{c}\right)^{2} j^{2}\right], \quad \epsilon^{(a)}=\int \frac{d V}{8 \pi} h^{2} .
$$

Then, for the potential gauged to zero at $\infty$ (which is possible in zero applied field) one has

$$
8 \pi \epsilon^{(a)}=\oint \varphi \mathbf{h} \cdot d \mathbf{S},
$$

where the integral is over the sample's surface with $d \mathbf{S}$ directed inward the material. If (as it may happen in some situations considered below) $\varphi$ does not vanish at infinity, one has to examine the integral (9) at infinity.

The London part can be transformed integrating the kinetic term by parts:

$$
8 \pi \epsilon^{(i)}=\frac{4 \pi \lambda^{2}}{c} \oint(\mathbf{h} \times \mathbf{j}) \cdot d \mathbf{S},
$$

where the integral is over the samples surface and the surface of the vortex core; there might be more than one sample in the system, while not all of them may contain vortices. The integral over the samples surface is further transformed:

$$
\oint d \mathbf{S} \cdot(\mathbf{j} \times \nabla \varphi)=\oint d \mathbf{S} \cdot \varphi(\nabla \times \mathbf{j})
$$

(take a closed contour at the sample surface, consider the total sample surface as made of two pieces supported by this contour, and apply the Stokes theorem to the integration over each piece to show that $\oint d \mathbf{S} \cdot(\nabla \times \varphi \mathbf{j})=0)$. Combining the result with $\epsilon^{(a)}$ of (9), one obtains

$$
\oint d \mathbf{S} \cdot \varphi\left(\mathbf{h}+\frac{4 \pi \lambda^{2}}{c} \operatorname{curl} \mathbf{j}\right) .
$$

The expression in parentheses is zero for samples with no vortices, whereas for those containing vortices it is $\phi_{0} \hat{\mathbf{v}} \delta^{(2)}\left(\mathbf{r}-\mathbf{r}_{v}\right)$ where $\hat{\mathbf{v}}$ is the direction of the vortex crossing the surface at the point $\mathbf{r}_{v}\left(\delta^{(2)}\left(\mathbf{r}-\mathbf{r}_{v}\right)\right.$ is the two-dimensional $\delta$ function). We then obtain

$$
\frac{8 \pi}{\phi_{0}} \epsilon=\varphi\left(\mathbf{r}_{e n t}\right)-\varphi\left(\mathbf{r}_{e x}\right)-\frac{4 \pi \lambda^{2}}{\phi_{0} c} \oint_{\text {core }} d \mathbf{S} \cdot(\mathbf{h} \times \mathbf{j}),
$$


with $\mathbf{r}_{e n t}$ and $\mathbf{r}_{e x}$ being the positions of the vortex entry and exit at the sample surface (the vortex is assumed to cross the sample surface at right angles). Note that if there are more than one superconductor present, but the vortex pierces only one of them, the result (11) still holds (although $\varphi$ 's differ for each particular configuration). For more than one vortex in the system, one has to sum up over all vortices. For thin films, the integral over the core surface $(\propto d)$ can be neglected in Eq. (11).

It is instructive to see now how the known Pearl result, 10,11 for vortex in a film and a bulk half-space can be obtained within the approach outlined here. We also demonstrate an added advantage of the method, a relatively simple way to evaluate energies.

\section{A. Vortex in a thin film}

Consider a thin film situated at $z=0$. The general form of the potential which vanishes at $z \rightarrow+\infty$ of the empty upper half-space is

$$
\varphi(\mathbf{r}, z>0)=\int \frac{d^{2} \mathbf{k}}{(2 \pi)^{2}} \varphi_{u}(\mathbf{k}) e^{i \mathbf{k} \cdot \mathbf{r}-k z} .
$$

with $k=\sqrt{k_{x}^{2}+k_{y}^{2}}$. In the lower half-space we have to replace $z \rightarrow-z$ in Eq. (12). The two dimensional (2D) Fourier transforms $\varphi_{u}(\mathbf{k})$ and $\varphi_{l}(\mathbf{k})$ for the upper and lower half-spaces are obtained with the help of boundary conditions (6.6):

$$
-k \varphi_{u}+\Lambda k^{2}\left(\varphi_{l}-\varphi_{u}\right)=\phi_{0}, \quad-k \varphi_{u}=k \varphi_{l} .
$$

This system yields:

$$
\varphi_{u}=-\frac{\phi_{0}}{k(1+2 k \Lambda)} .
$$

We point out first that the total flux crossing a plane $z=$ const is

$$
\Phi_{z}=\int h_{z} d^{2} \mathbf{r}=h_{z}(\mathbf{k}=0)=-\left.k \varphi_{u} e^{-k z}\right|_{k=0}=\phi_{0}
$$

for any $z$, i.e., the film is crossed by the flux $\phi_{0}$. And the second: according to (11), the energy $\epsilon$ of the Pearl vortex is given by

$$
\begin{aligned}
\frac{8 \pi \epsilon}{\phi_{0}} & =\varphi_{l}(0)-\varphi_{u}(0)=-2 \varphi_{u}(0)=\int \frac{d^{2} \mathbf{k}}{(2 \pi)^{2}} \frac{2 \phi_{0}}{k(1+2 k \Lambda)} \\
& =\frac{\phi_{0}}{\pi} \int_{0}^{2 \pi / \xi} \frac{d k}{1+2 k \Lambda},
\end{aligned}
$$

where the cutoff at $k_{\max } \approx 2 \pi / \xi$ is introduced to a logarithmically divergent integral. This yields

$$
\epsilon=\frac{\phi_{0}^{2}}{16 \pi^{2} \Lambda} \ln \left(4 \pi \frac{\Lambda}{\xi}\right) .
$$

\section{B. Vortex in a half-space}

Let now the half-space $z<0$ be filled with a superconductor having the penetration depth $\lambda$. The stray field in the free space $z>0$ is given by the potential (12). Within the superconductor we have the London equation (3). The general solution can be written as

$$
\mathbf{h}=\mathbf{h}^{(0)}+\mathbf{h}^{(v)},
$$

where $\mathbf{h}^{(0)}$ solves Eq. (3) with zero right-hand side (RHS), whereas $\mathbf{h}^{(v)}$ is a particular solution of the full Eq. (3). The latter can be taken as the field of an infinitely long unperturbed vortex along $z$; this assures correct singular behavior at the vortex axis. The Fourier transform of this field is

$$
\mathbf{h}^{(v)}=\frac{\phi_{0}}{1+\lambda^{2} k^{2}} \hat{\mathbf{z}}
$$

We now Fourier transform the homogeneous Eq.(3) for $h_{i}^{(0)}(\mathbf{r})$ with respect to the variable $\mathbf{r}$ and obtain

$$
h_{i}^{(0)}(\mathbf{k}, z)\left(1+k^{2} \lambda^{2}\right)-\lambda^{2} \partial_{z}^{2} h_{i}^{(0)}(\mathbf{k}, z)=0,
$$

where $i=x, y, z$. The solution which vanishes deep in the superconductor is

$$
h_{i}^{(0)}(\mathbf{k}, z)=H_{i}(\mathbf{k}) e^{q z}, \quad q=\sqrt{1+\lambda^{2} k^{2}} / \lambda .
$$

Here, $H_{i}(\mathbf{k})$ 's are not independent: $\operatorname{div} \mathbf{h}^{(0)}=0$ gives

$$
i k_{x} H_{x}+i k_{y} H_{y}+q H_{z}=0 .
$$

The boundary conditions at $z=0$ read:

$$
\begin{aligned}
i k_{x, y} \varphi_{u} & =H_{x, y}, \\
-k \varphi_{u} & =H_{z}+h_{z}^{(v)} .
\end{aligned}
$$

The four Eqs. (22, 24) suffice to determine the four unknowns, $\varphi_{u}$ and $H_{x, y, z}$. We obtain

$$
\varphi=-\frac{\phi_{0}}{k q(k+q) \lambda^{2}}, \quad H_{z}=-\frac{\phi_{0} k}{q^{2}(q+k) \lambda^{2}} .
$$

We do not write down $H_{x, y}$ which describe the spreading of the vortex field under the surface. Equations (25) coincide with Pearl's solution.11

To evaluate the energy of the vortex in this case with the help of the general result (11), we first calculate the potential at the vortex exit:

$$
\varphi(0)=\int \frac{d^{2} \mathbf{k}}{(2 \pi)^{2}} \varphi(\mathbf{k})=-\frac{\phi_{0}}{2 \pi \lambda} .
$$

The energy associated with the core, i.e., the integral over the core surface in Eq. (11), is 


$$
\frac{2 c}{\lambda^{2}} \epsilon_{c}=2 \pi \xi \int_{-\infty}^{0} d z\left[h_{z} j_{\theta}\right]_{r=\xi}
$$

Integrating the London relation

$$
j_{\theta}=-\frac{c \phi_{0}}{2 \pi^{2} \lambda^{2}}\left(\nabla \theta+\frac{2 \pi}{\phi_{0}} \mathbf{A}\right)_{\theta}
$$

over a circle of a radius $r$, we obtain for the current density near the core

$$
j_{\theta}=\frac{c \phi_{0}}{8 \pi^{2} \lambda^{2} r}
$$

which results in

$$
\epsilon_{c}=\frac{\phi_{0}}{8 \pi} \int_{-\infty}^{0} d z h_{z}
$$

Using $H_{z}$ of Eq. 25 with Eqs. (11) and (26) we obtain the energy cost of vortex termination at the surface as

$$
\frac{\phi_{0}^{2}}{16 \pi^{2} \lambda}\left(\frac{\pi}{2}-1\right) \approx \frac{0.57}{\ln \kappa} \epsilon_{L} \lambda
$$

where $\epsilon_{L}$ is the energy per unit length of an unperturbed vortex.

\section{SUBSURFACE TERMINATION}

\section{A. Half-space + a thin layer}

We now turn to a stack of pancake vortices perpendicular to the layers of a half-space layered sample. The layered structure has the period $s$ whereas each layer is characterized by the Pearl length $\Lambda=\lambda^{2} / d$ with $d$ being the layer thickness. Note that the measurable length $\lambda_{a b}$ is related to other lengths in the problem by

$$
\lambda_{a b}^{2}=\lambda^{2} \frac{s}{d}=\Lambda s
$$

Let us consider first the stack with a missing pancake in the top layer. We model the rest of the stack as a continuous half-space $z<0$ with the penetration depth $\lambda_{a b}$. A thin film with the Pearl length $\Lambda$ is situated at $z=s$. In the two domains of free space, $z>s$ and $0<z<s$, the 2D Fourier transforms of the potential are:

$$
\begin{array}{cr}
\varphi_{u}(\mathbf{k}) e^{-k z}, & z>s, \\
\varphi_{1}(\mathbf{k}) e^{k z}+\varphi_{2}(\mathbf{k}) e^{-k z}, & 0<z<s .
\end{array}
$$

On the film at $z=s$, we have the boundary conditions (7) and (6). Three more equations are provided by the field continuity at $z=0$. The requirement $\operatorname{div} \mathbf{h}^{(0)}=0$, Eq. (22), completes the system thus providing enough equations for $\varphi_{u}, \varphi_{1,2}$ and $H_{x, y, z}$ :

$$
\begin{aligned}
\varphi_{u} & =\frac{2 \phi_{0} \Lambda e^{2 k s}}{D q \lambda_{a b}^{2}}, \quad \varphi_{1}=\frac{\phi_{0}}{D q k \lambda_{a b}^{2}} \\
\varphi_{2} & =\frac{\phi_{0} e^{2 k s}}{D q k \lambda_{a b}^{2}}(1+2 k \Lambda) \\
D & =q-k-(1+2 k \Lambda)(k+q) e^{2 k s}
\end{aligned}
$$

and

$$
H_{z}(\mathbf{k})=\frac{\phi_{0}}{D q \lambda_{a b}^{2}}\left[1-e^{2 k s}(1+2 k \Lambda)\right]-\frac{\phi_{0}}{q^{2} \lambda_{a b}^{2}},
$$

where $q$ is defined in Eq. (21); $H_{x, y}$ is not needed here.

The fluxes through planes $z=$ const are:

$$
\begin{aligned}
& \Phi_{z}=\phi_{0} \frac{\Lambda}{\Lambda+\lambda_{a b}}, \quad z>s, \\
& \Phi_{z}=\phi_{0}, \quad z<0 \text {. }
\end{aligned}
$$

Within the layer $0<z<s$, the flux $\Phi_{z}$ decreases from the bulk value $\phi_{0}$ to the value (37). Therefore, a small fraction of the flux,

$$
\phi_{0} \lambda_{a b} / \Lambda=\phi_{0} s / \lambda_{a b},
$$

is channelled aside between the half-space and the top film.

Using the same formal scheme, we can solve the problem when the film at $z=s$ contains a pancake vortex. The only difference with the case of "half-space + an empty film" is in the London boundary condition (7) on the film at $z=s$. We skip details and provide the result denoting the new solutions with a star:

$$
\begin{aligned}
\varphi_{u}^{*} & =\varphi_{u}+\frac{\phi_{0} e^{k s}}{k D}\left[k-q+(k+q) e^{2 k s}\right], \\
\varphi_{1,2}^{*} & =\varphi_{1,2} \mp \frac{\phi_{0} e^{k s}}{D k}(q \pm k), \\
H_{z}^{*} & =H_{z}-\frac{2 k \phi_{0}}{D} .
\end{aligned}
$$

The energy cost of vortex termination, i.e., the difference between the situations without and with the top pancake, is given by

$$
\begin{aligned}
\frac{8 \pi}{\phi_{0}} \Delta \epsilon & =\left[-\varphi_{e x}(0)\right]-\left[\varphi_{\text {ent }}^{*}(s)-\varphi_{e x}^{*}(s)-\varphi_{e x}^{*}(0)\right] \\
& +\int_{-\infty}^{0} d z\left(h_{z}-h_{z}^{*}\right)
\end{aligned}
$$

the last line is the difference in core contributions of vortex lines in the low half-space, see Eq. (30)

Evaluating the Fourier integrals for $\varphi$ 's, one notes that the integration over $k$ is done within the domain $2 \pi / R, 2 \pi / \xi$ where $R$ is the sample size and $\xi$ is the coherence length (usually called $\xi_{a b}$ ). For $s<\xi$ one can expand $e^{-2 k s}$ in the denominator $D$ and see that the term with $s$ can be neglected. Indeed, by introducing a new variable $\sinh u=k \lambda_{a b}$ and utilizing $\Lambda \gg \lambda_{a b}$, we obtain 


$$
D \approx-2 k\left[1+\left(\Lambda / \lambda_{a b}\right) e^{u}\right] \approx-2 k e^{u} \Lambda / \lambda_{a b} .
$$

This results in a factor $1 / \Lambda=s / \lambda_{a b}^{2}$ in front of the integrals. Then, in the linear approximation in $s$, we can set $s=0$ in the integrand after which all integrals become simple. We obtain after a straightforward algebra:

$$
\Delta \epsilon=\frac{\phi_{0}^{2}}{16 \pi^{2} \Lambda} \ln \left[\frac{R}{(4 \pi)^{3 / 2} \kappa \lambda_{a b}}\right] .
$$

It is of interest to obtain the current distribution in the top film. To this end, we write Eq. (12) in cylindric coordinates:

$$
\varphi(\mathbf{r})=\int \frac{d k k}{2 \pi} J_{0}(k r) \varphi(\mathbf{k}) e^{-k z} .
$$

The tangential fields above and under the film are:

$$
\begin{aligned}
& h_{r}^{+}=-\int_{0}^{\infty} \frac{d k}{2 \pi} k^{2} J_{1}(k r) \varphi_{u} e^{-k s}, \\
& h_{r}^{-}=-\int_{0}^{\infty} \frac{d k}{2 \pi} k^{2} J_{1}(k r)\left(\varphi_{1} e^{k s}+\varphi_{2} e^{-k s}\right) .
\end{aligned}
$$

The sheet current is given by

$$
\frac{4 \pi}{c} g_{\varphi}=h_{r}^{+}-h_{r}^{-}=2 \int_{0}^{\infty} \frac{k^{2} d k}{2 \pi} J_{1}(k r) \varphi_{1} e^{k s},
$$

where the boundary condition (耳) at $z=s, \varphi_{u}+\varphi_{1} e^{2 k s}-$ $\varphi_{2}=0$, had been used. With the help of Eq. (42) for $D$, we obtain after integration (see Ref. 13, 6.663):

$$
g_{\varphi}=\frac{c \phi_{0}}{8 \pi^{2} \Lambda \lambda_{a b}} I_{1}\left(\frac{r}{2 \lambda_{a b}}\right) K_{0}\left(\frac{r}{2 \lambda_{a b}}\right)
$$

where $I_{1}, K_{0}$ are Modified Bessel functions. Thus, $g_{\varphi}$ vanishes as $r \ln \left(2 \lambda_{a b} / r\right)$ when $r \rightarrow 0$, and as $1 / r$ for $r \gg \lambda_{a b}$.

We note that the same formal scheme can be applied to consider the termination of the pancake stack in the second, third, etc, layer from the top. The length $\Lambda$ characterizing the top layers with no cores should then be taken as $\Lambda / 2, \Lambda / 3$, etc.

\section{B. Vortex in a finite stack of layers}

As an example of such a system we consider a pancake in middle layer of a "short stack" of three films. Let the pancake sit at the origin of the film at $z=0$; the film is characterized by the length $\Lambda$. At $z \pm s$ two other films are situated having the film penetration depth $\Lambda_{1} \neq \Lambda$. Following the same method we write the $2 \mathrm{D}$ Fourier transforms:

$$
\begin{array}{lc}
\varphi_{a} e^{-k z}, & z>s, \\
\varphi_{b 1} e^{k z}+\varphi_{b 2} e^{-k z}, & 0<z<s, \\
\varphi_{c 1} e^{k z}+\varphi_{c 2} e^{-k z}, & -s<z<0 .
\end{array}
$$

Due to the symmetry, $h_{b z}(z)=h_{c z}(-z)$, and $\varphi_{c 1}=$ $-\varphi_{b 2}, \varphi_{c 2}=-\varphi_{b 1}$; this leaves only three coefficients $\varphi$ to be determined. The boundary conditions (7) and (6) at $z=s$ give

$$
\begin{aligned}
\varphi_{a}+\varphi_{b 1} e^{2 k s}-\varphi_{b 2} & =0, \\
\varphi_{a}\left(1+k \Lambda_{1}\right)-k \Lambda_{1}\left(\varphi_{b 1} e^{2 k s}+\varphi_{b 2}\right) & =0 .
\end{aligned}
$$

The third equation is provided by the London boundary condition (6) at $z=0$ :

$$
\varphi_{b 1}(1-2 k \Lambda)-\varphi_{b 2}(1+2 k \Lambda)=\phi_{0} / k .
$$

The continuity condition (7) for $h_{z}$ at $z=0$ is satisfied identically. Equations (49) 51) yield

$$
\begin{aligned}
\varphi_{a} & =\frac{2 \phi_{0} \Lambda_{1}}{D_{1}}, \quad \varphi_{b 1}=\frac{\phi_{0}}{k D_{1}} e^{-2 k s} \\
\varphi_{b 2} & =\frac{\phi_{0}}{k D_{1}}\left(1+2 k \Lambda_{1}\right), \\
D_{1} & =(1-2 k \Lambda) e^{-2 k s}-(1+2 k \Lambda)\left(1+2 k \Lambda_{1}\right) .
\end{aligned}
$$

The fluxes are

$$
\begin{aligned}
\Phi_{z}(z>s) & =\phi_{0} \frac{\Lambda_{1}}{2 \Lambda+\Lambda_{1}+s} \\
\Phi_{z}(0<z<s) & =\phi_{0} \frac{\Lambda_{1}+s-z}{2 \Lambda+\Lambda_{1}+s} .
\end{aligned}
$$

Note that the flux $\phi_{0} s /\left(2 \Lambda+\Lambda_{1}\right) \ll \phi_{0}$ is deflected into the space between the layers. For $\Lambda \gg \Lambda_{1} \gg s$,

$$
\Phi_{z}=\phi_{0} \frac{\Lambda_{1}}{2 \Lambda} .
$$

Note also that for $\Lambda_{1}=\Lambda \gg s$, the flux piercing the sandwich made of identical films is $\phi_{0} / 3$. The energy is given by

$$
\frac{8 \pi \epsilon}{\phi_{0}}=\left[\varphi_{c}-\varphi_{b}\right]_{\mathbf{r}=z=0}=-2 \int \frac{d^{2} \mathbf{k}}{(2 \pi)^{2}}\left(\varphi_{b 1}+\varphi_{b 2}\right) .
$$

Using the same argument as that leading to Eq. (42), we obtain for the denominator

$$
D_{1} \approx-2 k \Lambda\left(\frac{2 \Lambda+\Lambda_{1}}{\Lambda}+2 k \Lambda_{1}\right) .
$$

Integration in Eq. (55) is now easy.

Consider the case $\Lambda_{1}=\Lambda$ and set $s=0$ (everywhere except in $\Lambda=\lambda^{2} / s$ ). Integration yields for the energy of one pancake in a stack of three films

$$
\epsilon_{010} \approx \frac{\phi_{0}^{2} / 3}{16 \pi^{2} \Lambda} \ln \frac{16 \pi^{2} \Lambda^{2} R}{9 \xi^{3}} .
$$

where it was assumed that the sample size $R \gg 2 \pi \Lambda$ and the subscript 010 is to indicate that the pancake is situated in the middle film. This is to be compared with a vortex piercing all three layers, which can be considered 
as a Pearl vortex in a single film with the effective film penetration depth $\Lambda / 3$. Then, according to Eq. (17),

$$
\epsilon_{111}=\frac{3 \phi_{0}^{2}}{16 \pi^{2} \Lambda} \ln \frac{4 \pi \Lambda}{3 \xi},
$$

and the subscript 111 indicates that each layer has a pancake. The difference of energies (57) and (58) is negative if

$$
R<\Lambda(\Lambda / \xi)^{6}
$$

and we have omitted a large numerical factor. This suggests that in the stack of three films, vortices carrying the flux $\phi_{0} / 3$ with a core only in the middle film might be energetically preferable to standard vortices piercing the whole stack with the flux $\phi_{0}$.

It is worth noting that because our solution holds for $\Lambda \neq \Lambda_{1}$, in fact, it applies also to a vortex piercing $N$ middle layers of the $2 N_{1}+N$ stack of layers provided that both $N_{1} s$ and $N s$ are less than $\lambda_{a b}$; this allows us to treat the $N_{1} s$ as one film with $\Lambda_{1}=\lambda_{a b}^{2} / N_{1} s$.

\section{INFINITE DILUTE STACK OF PANCAKES}

We consider now an infinite stack of layers. Let the layer at $z=0$ and all even layers be free of pancakes; the pancakes are situated at $z= \pm s$ and at all odd films (at $\mathbf{r}=0$ ). Consider three regions: (a) $s<z<2 s$, (b) $0<z<s$, and (c) $-s<z<0$.

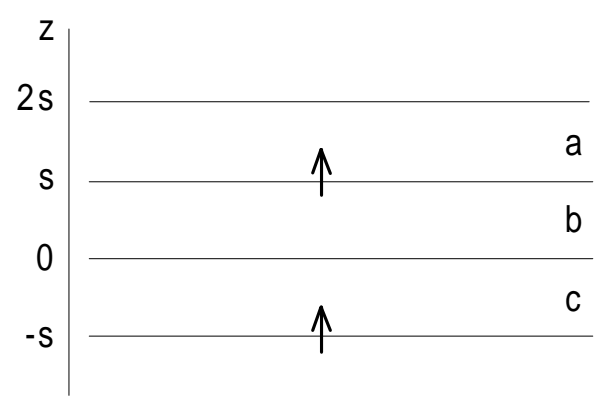

FIG. 1. The unit cell of a periodic structure $0<z<2 s$.

The 2D Fourier transforms:

$$
\begin{aligned}
\varphi_{a} & =\varphi_{a 1} e^{k z}+\varphi_{a 2} e^{-k z} \\
\varphi_{b} & =\varphi_{b 1} e^{k z}+\varphi_{b 2} e^{-k z} \\
\varphi_{c} & =\varphi_{c 1} e^{k z}+\varphi_{c 2} e^{-k z}
\end{aligned}
$$

Due to the symmetry with respect to $z=0, h_{b z}(z)=$ $h_{c z}(-z)$. This gives $\varphi_{c 1}=-\varphi_{b 2}, \varphi_{c 2}=-\varphi_{b 1}$. Further relations are provided by periodicity which can be expressed as $h_{a z}(z)=h_{c z}(z-2 s)$ and yields

$$
\varphi_{a 1}=-\varphi_{b 2} e^{-2 k s}, \quad \varphi_{a 2}=-\varphi_{b 1} e^{2 k s} .
$$

Thus, out of 6 unknown coefficients of the system (60-62) we are left with only two. As the two needed equation one can take the London conditions (6) at $z=0$ and $z=s$ :

$$
\begin{aligned}
\varphi_{b 1}(1-2 k \Lambda)-\varphi_{b 2}(1+2 k \Lambda) & =0, \\
\varphi_{b 1} e^{k s}(1+2 k \Lambda)-\varphi_{b 2} e^{-k s}(1-2 k \Lambda) & =\phi_{0} / k .
\end{aligned}
$$

This gives

$$
\begin{aligned}
\varphi_{b 1} & =\frac{\phi_{0}}{k D_{2}}(1+2 k \Lambda), \\
\varphi_{b 2} & =\frac{\phi_{0}}{k D_{2}}(1-2 k \Lambda), \\
D_{2} & =e^{k s}(1+2 k \Lambda)^{2}-e^{-k s}(1-2 k \Lambda)^{2} .
\end{aligned}
$$

The flux at any plane $z=$ const within the domain (b) is readily evaluated:

$$
\begin{aligned}
\Phi_{b}(z) & =h_{b z}(k=0)=\left[k \varphi_{b 1} e^{k z}-k \varphi_{b 2} e^{-k z}\right]_{k=0} \\
& =\phi_{0} \frac{2 \Lambda+z}{4 \Lambda+s} \approx \frac{\phi_{0}}{2} .
\end{aligned}
$$

Similarly, in the domain (c):

$$
\Phi_{c}(z)=\phi_{0} \frac{2 \Lambda-z}{4 \Lambda+s} \approx \frac{\phi_{0}}{2} .
$$

Therefore, $\Phi \approx \phi_{0} / 2$ everywhere throughout the system.

Since we have in this case only one pancake per the period $2 s$ of the structure, the line energy of the stack $\epsilon=\epsilon_{p} / 2 s$ where $\epsilon_{p}$ is the energy per pancake:

$$
\begin{aligned}
\frac{8 \pi \epsilon_{p}}{\phi_{0}} & =\varphi_{b}(s)-\varphi_{a}(s)=2 \varphi_{b}(s) \\
& =2 \int \frac{d^{2} \mathbf{k}}{(2 \pi)^{2}}\left(\varphi_{b 1} e^{k s}+\varphi_{b 2} e^{-k s}\right) .
\end{aligned}
$$

As above, within the integration domain we can simplify the denominator, $D_{2} \approx 8 k \Lambda$, and set $s=0$ in the integrand. Then we obtain after integration within $2 \pi / R, 2 \pi / \xi$ :

$$
\epsilon=\left(\frac{\phi_{0}}{8 \pi \lambda_{a b}}\right)^{2} \ln \frac{R}{\xi} .
$$

Comparing this with the line energy of a standard vortex $\left(\phi_{0} / 4 \pi \lambda_{a b}\right)^{2} \ln \kappa$ we see that dilute stacks considered here are possible in small samples (whiskers) of a size $R<\lambda_{a b} \kappa^{3}$.

\section{DISCUSSION}

We now estimate how the energy cost 43 ) of the subsurface vortex termination is affected by the Josephson coupling. Clearly, this coupling breaks the cylindrical symmetry of the field associated with the straight stack of pancake vortices, and the flux (39) will not be spread even in all directions in the space between the top layer 
and the rest. Still, for small samples $R<\lambda_{J}$, in which the string cannot fully develop, the asymmetry can be disregarded. Since the phase difference is zero for perfectly aligned stack of pancakes for all pairs of layers except the top one, the Josephson energy can be estimated as

$$
\begin{aligned}
\epsilon_{J} & =\frac{\phi_{0}^{2}}{8 \pi^{3} s \lambda_{c}^{2}} \int_{0}^{R} r d r \int_{0}^{2 \pi} d \theta(1-\cos \theta) \\
& \approx 2 s\left(\frac{\phi_{0} R}{4 \pi \lambda_{a b} \lambda_{J}}\right)^{2}=\frac{\phi_{0}^{2}}{8 \pi^{2} \Lambda} \frac{R^{2}}{\lambda_{J}^{2}} .
\end{aligned}
$$

On the other hand, for $R \gg \lambda_{J}$, the integral in (70) is roughly proportional to the string area $R \lambda_{J}$ because $\lambda_{J}$ is an estimate for the string width:

$$
\epsilon_{J}=\frac{\phi_{0}^{2}}{8 \pi^{3} s \lambda_{c}^{2}} R \lambda_{J}=\frac{\phi_{0}^{2}}{8 \pi^{3} \Lambda} \frac{R}{\lambda_{J}} .
$$

The energies $(70)$ or $(71)$ should be added to the estimate (43) for the energy cost of the subsurface vortex termination.

Taking, for example, Bi-2212 with $s=15 \AA, \lambda_{J}=$ $4500 \AA$, $\kappa=\lambda_{a b} / \xi_{a b}=50$, we have for $R=1 \mu$ :

$$
\Delta \epsilon=\frac{\phi_{0}^{2}}{16 \pi^{2} \Lambda}\left[\ln \left(\frac{R}{\kappa \lambda_{a b}(4 \pi)^{3 / 2}}\right)+\frac{2 R}{\pi \lambda_{J}}\right] .
$$

We estimate the logarithm here as $\approx-2$ (disregarding the factor $(4 \pi)^{-3 / 2}$ which could reduce this estimate to $-4)$. The Josephson correction is $2 R / \pi \lambda_{J} \approx 1.4$. Thus, in micron-size samples of Bi-2212, the termination of vortices under the surface is possible.

The above treatment is concerned with the case of zero applied field. The energy cost of the subsurface termination should increase with increasing applied field. To have an estimate of this increase, one can evaluate the work needed to remove a pancake from the top layer of a cylindrical samle of radius $R$ in the presence of a small external field $H_{a}$. Since one layer screening ability is weak, we assume the field uniform, which corresponds to the asymuthal current density $j=c A / 4 \pi \lambda^{2}=c H_{a} r / 8 \pi \lambda^{2}$ (the vector potential can be taken as $A=H_{a} r / 2$ ). The work of the Lorentz force to remove a pancake from the center is now readily obtained as $\approx H_{a} \phi_{0} R^{2} / 16 \pi \Lambda$. To compare with the estimate 72 ) we write the extra cost due to the applied field as

$$
\epsilon_{a}=\frac{\phi_{0}^{2}}{16 \pi^{2} \Lambda} \frac{H_{a} \pi R^{2}}{\phi_{0}} .
$$

Since the expression in parentheses of Eq. 72 is on the order one, we can say that the energy gain of the subsurface termination is lost in fields $H_{a} \sim \phi_{0} / \pi R^{2}$ which gives $H_{a} \sim 10 \mathrm{G}$ for $R=1 \mu$. This sets an approximate upper bound on fields in which the subsurface termination may occur.

We would like to mention in conclusion that, since configurations considered here and similar ones are not forbidden by topology, they should be taken into account while studying fluctuations in layered weakly coupled materials. They are certainly of interest for physics of layered organic superconductors in which $\lambda_{c}$ may reach a tens-of-micron size at low temperatures (see, e.g., Ref. 2) as well as for superconducting multilayers where the Josephson coupling can be reduced by varying the interlayer spacing.

\section{ACKNOWLEDGMENTS}

This research was supported by grant No. 96-00048 from the United States - Israel Binational Science Foundation (BSF), Jerusalem, Israel. The work at Ames was supported by the Office of Basic Energy Sciences, U.S. Department of Energy.

${ }^{1}$ J. C. Martnez, S. H. Brongersma, A. Koshelev, B. Ivlev, P. H. Kes, R. P. Griessen, D. G. de Groot, Z. Tarnavski, and A. A. Menovsky Phys. Rev. Lett. 69, 2276 (1992).

2 J.R. Kirtley, K.A. Moler, J.A. Schlueter, and J.M. Williams, J. Phys. Condens. Matt. 11, 2007 (1999).

${ }^{3}$ K.B. Efetov, Zh. Eksp. Teor. Fiz. 76, 1781 (1979).

${ }^{4}$ F. Guinea, Phys. Rev. B 426244 (1990).

${ }^{5}$ A. Buzdin and D. Feinberg, J. Phys. France 511971 (1990).

${ }^{6}$ N.S. Artemenko and A.N. Kruglov, Phys. Lett. A 143485 (1990).

7 J. R. Clem, Phys. Rev. B 437837 (1991).

${ }^{8}$ G. Blatter, M.V. Feigel'man, V.B. Geshkenbein, A.I. Larkin, V.M. Vinokur, Rev. Mod. Phys. 66, 1125 (1994).

${ }^{9}$ V. G. Kogan, Phys. Rev. B 49, 15874 (1994).

10 J. Pearl, Appl. Phys. Lett 5, 65 (1964).

11 J. Pearl, J. Appl. Phys. 37, 4139 (1966).

${ }^{12}$ Handbook of Mathematical Functions with Formulas, Graphs, and Mathematical Tables, edited by M. Abramowitz and A. Stegun, Natl. Bur. Stand. Appl. Math. Ser. No. 55 (U.S. GPO, Washington, D.C., 1965).

${ }^{13}$ I. S. Gradshteyn and I. M. Ryzhik, Tables of Integrals, Series, and Products, Academic Press, 1980. 